\title{
Papers
}

\section{Randomised controlled trial of exercise for low back pain: clinical outcomes, costs, and preferences}

Jennifer Klaber Moffett, David Torgerson, Sally Bell-Syer, David Jackson, Hugh Llewlyn-Phillips, Amanda Farrin, Julie Barber

\begin{abstract}
Objective To evaluate effectiveness of an exercise programme in a community setting for patients with low back pain to encourage a return to normal activities.

Design Randomised controlled trial of progressive exercise programme compared with usual primary care management. Patients' preferences for type of management were elicited independently of randomisation.

Participants 187 patients aged 18-60 years with mechanical low back pain of 4 weeks to 6 months' duration.

Interventions Exercise classes led by a

physiotherapist that included strengthening exercises for all main muscle groups, stretching exercises, relaxation session, and brief education on back care. A cognitive-behavioural approach was used.

Main outcome measures Assessments of debilitating effects of back pain before and after intervention and at 6 months and 1 year later. Measures included Roland disability questionnaire, Aberdeen back pain scale, pain diaries, and use of healthcare services. Results At 6 weeks after randomisation, the intervention group improved marginally more than the control group on the disability questionnaire and reported less distressing pain. At 6 months and 1 year, the intervention group showed significantly greater improvement in the disability questionnaire score (mean difference in changes $1.35,95 \%$ confidence interval 0.13 to 2.57 ). At 1 year, the intervention group also showed significantly greater improvement in the Aberdeen back pain scale (4.44, 1.01 to 7.87 ) and reported only 378 days off work compared with 607 in the control group. The intervention group used fewer healthcare resources. Outcome was not influenced by patients' preferences.

Conclusions The exercise class was more clinically effective than traditional general practitioner management, regardless of patient preference, and was cost effective.
\end{abstract}

\section{Introduction}

Low back pain is common and, although it may settle quickly, recurrence rates are about $50 \%$ in the following 12 months. ${ }^{1}$ Recent management guidelines recommend that an early return to physical activities should be encouraged, ${ }^{2}{ }^{3}$ but patients are often afraid of movement after an acute onset of back pain. Trials of specific exercise programmes for acute back pain have not shown them to be effective, ${ }^{45}$ but a specific exercise programme may have to be tailored to suit the individual patient and so is less likely to be effective for a heterogeneous group of patients.

However, there is some evidence that a general exercise programme, which aims to increase individuals' confidence in the use of their spine and overcome the fear of physical activity, can be effective for patients with chronic back pain (of more than six months' duration). A recent randomised trial of a supervised exercise programme in a hospital setting reported significantly better outcomes at six months and two years for the exercise group compared with the control group. ${ }^{67}$ Whether this approach would be effective and cost effective for patients with low back pain of less than six months' duration in a primary care setting is unknown.

An important methodological problem occurs when it is not possible to blind subjects to the treatment they receive, since outcome is probably directly influenced by their preconceived ideas regarding the effectiveness of intervention. ${ }^{8}$ Thus, in trials where a double blind procedure is not feasible, participants who are not randomised to their treatment of choice may be disappointed and suffer from resentful demoralisation, ${ }^{9}$ whereas those randomised to their preferred treatment may have a better outcome irrespective of the physiological efficacy of the intervention. However, this problem may be partly ameliorated if patients' treatment preferences are elicited before randomisation, so that they can be used to inform the analysis of costs and outcomes..$^{10} 11$

In this paper, we report a fully randomised trial for the treatment of subacute low back pain in which the analysis was informed by patient preference.

\section{Subjects and methods}

\section{Recruitment of subjects}

Eighty seven general practitioners agreed to participate in the study, and the principal investigator (JKM) visited each practice to discuss participation. Selection of general practitioners was based in the York area and

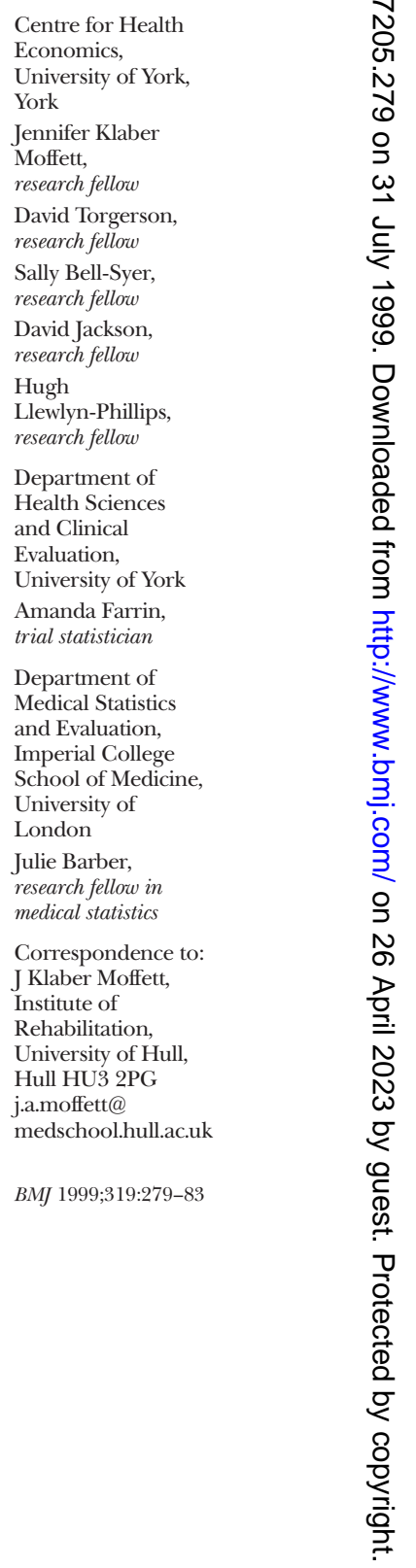


restricted by the need to provide easy access for patients to the classes. Only one invited practice declined to participate. Single handed practices were not invited. The general practitioners referred patients directly to the research team or sent a monthly list of patients who had consulted with back pain. Inclusion criteria were patients with mechanical low back pain of at least four weeks' duration but less than six months, aged between 18 and 60, declared medically fit by their general practitioner to undertake the exercise, and who had consulted one of the general practitioners participating in the study. Patients with any potentially serious pathology were excluded, as were any who would have been unable to attend or participate in the classes. The exclusion criteria were the same as described by Frost et $\mathrm{al}^{7}$ except that concurrent physiotherapy rather than previous physiotherapy was an exclusion criterion in this trial.

\section{Evaluation}

Patients who seemed eligible were contacted by telephone and if they were interested in participating in the study were invited to an initial interview, at which the study and its implications for participants were explained. Patients who met all the eligibility criteria and consented to participate attended a first assessment a week later.

This included a physical examination (to exclude possible serious spinal pathology) and collection of baseline data by means of validated measures of health status. The main outcome measures were the Roland back pain disability questionnaire, ${ }^{12}$ which measures functional limitations due to back pain, and the Aberdeen back pain scale, ${ }^{13}$ which is more a measure of clinical status. The Roland disability questionnaire consists of a 24 point scale: a patient scoring three points on the scale means that he or she reports, for example, "Because of my back I am not doing any of the jobs that I usually do around the house, I use a handrail to get upstairs, and I lie down to rest more often." We also administered the EuroQoL health index $(\mathrm{EQ}-5 \mathrm{D})^{14}$ and the fear and avoidance beliefs questionnaire $(\mathrm{FABQ}){ }^{15}$

The second assessment was carried out at the patients' general practice six weeks after randomisation to treatment. The brief physical examination was repeated, and the patients were asked to complete the same outcome questionnaires.

In addition, patients were asked to complete pain diaries in the week before their first assessment and in the week before their second assessment. The diaries were used to assess subjective pain reports and asked "How strong is the pain?" and "How distressing is the pain?"6 16

We also evaluated patients at six and 12 months' follow up by sending them outcome questionnaires to complete and return.

\section{Randomisation and treatments}

A pre-prepared randomisation list was generated from a random numbers table and participants were stratified by practice in blocks of six. The trial coordinator ensured concealment of allocation from the clinical researchers by providing the research physiotherapist with a sealed envelope for a named patient before baseline assessment. A note inside the envelope invited the participant either to attend exercise classes or to continue with the current advice or treatment offered by his or her general practitioner. (One of the referring general practitioners used manipulation as usual treatment on most of his patients so that up to 37 patients in each arm of the study could also have received manipulation.) Each patient had an equal chance of being allocated to the intervention or the control group. Before patients were given their envelope they were asked whether they had any preference for the treatment assignment. The participants opened the envelope after leaving the surgery.

Intervention group-The exercise programme consisted of eight sessions, each lasting an hour, spread out over four weeks, with up to 10 participants in each class. The programme was similar to the Oxford fitness programme $^{7}$ and included stretching exercises, low impact aerobic exercises, and strengthening exercises aimed at all the main muscle groups. The overall aim was to encourage normal movement of the spine. No special equipment was needed. Participants were discouraged from viewing themselves as invalids and from following the precept of "Let pain be your guide." They were encouraged to improve their individual record and were selectively rewarded with attention and praise. Although partly based on a traditional physiotherapy approach, the programme used cognitive-behavioural principles. One simple educational message encouraging self reliance was delivered at each class. Participants were told that they should regard the classes as a stepping stone to increasing their own levels of activity.

Controls-Patients allocated to the control group continued under the care of their doctor and in some cases were referred to physiotherapy as usual. No attempt was made to regulate the treatment they received, but it was recorded.

\section{Economic analysis}

We recorded patients' use of healthcare services using a combination of retrospective questionnaires and prospective diary cards, which they returned at 6 and 12 months' follow up. From this information we estimated the cost of each patient's treatment. We compared the mean costs of treatment for the two groups by using Student's $t$ tests and standard confidence intervals. However, as cost data were highly positively skewed, these results were checked with a nonparametric "bootstrap." 17 The economic evaluation addressed both costs to the NHS and the costs to society. Participants were not charged for the classes, in line with any treatment currently available on the NHS.

\section{Statistical analysis}

Our original intention was to recruit 300 patients, which, given a standard deviation of 4 , would have provided $90 \%$ power at the $5 \%$ significance level to detect a 1.5 point difference between the two groups in the mean change on the Roland disability questionnaire. However, recruitment of patients to the study proved much slower than expected, and, because of the limitations of study resources, recruiting was stopped after 187 patients had been included into the study. This smaller sample reduced the power to detect such a difference to $72 \%$, but there was still $90 \%$ power to detect a 2 point difference in outcome. 


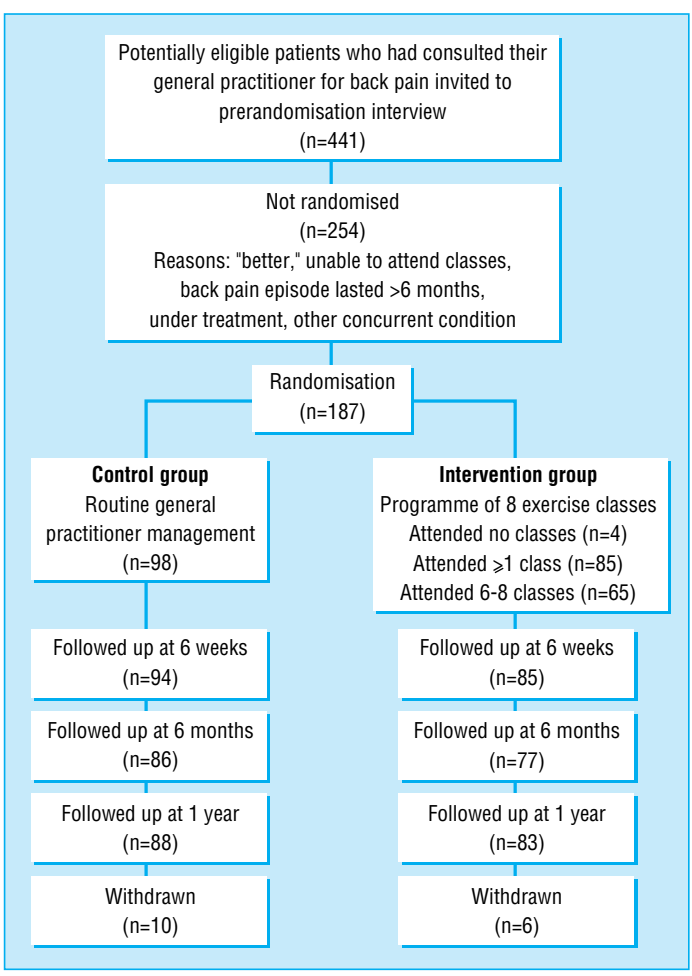

Flow chart describing patients' progress through the trial

Our analysis was based on intention to treat. We estimated the effects of treatment on the outcome measures by means of analysis of covariance, with the change in scores as the dependent variable and adjustment being made for baseline score and patient preference. We used Student's $t$ tests to analyse the data from the pain diaries as the baseline scores were quite similar.

\section{Results}

\section{Study population}

Of the 187 patients included in the trial, 89 were randomised to the intervention and 98 to the control group. The figure shows their progress through the trial. In both groups those with the most severe back pain at randomisation were less likely to return follow up questionnaires: the mean Roland disability questionnaire score for responders at one year follow up was 5.80 (SD 3.48) compared with a mean score of 9.06 (4.58) for non responders respectively $(\mathrm{P}=0.002)$.

\section{Baseline characteristics}

The clinical and demographic characteristics of the patients in the two groups were fairly well balanced at randomisation (table 1), although those allocated to the intervention group tended to report more disability on the Roland disability questionnaire than did the control group. Most patients (118, 63\%), when asked, would have preferred to be allocated to the exercise programme. Attendance of the classes was considered quite good, with $73 \%$ of the intervention group attending between six and eight of the classes. Four people failed to attend any classes and were included in the intention to treat analysis. No patients allocated to the control group took part in the exercise programme.

\section{Clinical outcomes}

Table 2 shows the mean changes in outcome measures over time, from randomisation to final follow up at one year. After adjustment for baseline scores, the intervention group showed greater decreases in all measures of back pain and disability compared with the controls. At six weeks after randomisation, patients in the intervention group reported less distressing pain than the control group $(\mathrm{P}=0.03)$ and a marginally significant difference on the Roland disability questionnaire scores. Other variables were not significantly different, but the differences in change were all in favour of the intervention group. At six months the difference of the mean change scores of the Roland disability questionnaire was significant, and at one year the differences in changes of both the Roland disability questionnaire and the Aberdeen back pain scale were significant (table 2). Most of the intervention group improved by at least three points on the Roland disability questionnaire: $53 \%$ (95\% confidence interval $42 \%$ to $64 \%)$ had

Table 1 Baseline characteristics of patients with mechanical low back pain included in study. Values are means (standard deviations) unless stated otherwise

\begin{tabular}{lcc} 
Variable & Control group (n=98) & $\begin{array}{c}\text { Intervention group } \\
(\mathbf{n}=89)\end{array}$ \\
\hline Age (years) & $42.6(8.62)$ & $41.1(9.21)$ \\
\hline No (\%) of women & $55(56)$ & $51(57)$ \\
\hline No (\%) of non-smokers & $69(70)$ & $64(72)$ \\
\hline No (\%) who preferred to be allocated to exercise & $65(66)$ & $53(60)$ \\
\hline No (\%) who had physiotherapy in past 6 months & $23(24)$ & $18(20)$ \\
\hline Number of visits to general practitioner in past 6 months & $2.45(2.36)$ & $2.22(3.32)$ \\
\hline Roland disability questionnaire score (0-24 points) & $5.56(3.94)$ & $6.65(4.02)$ \\
\hline Aberdeen back pain scale (0-100 points) & $25.52(10.85)$ & $27.93(11.07)$ \\
\hline Fear-avoidance beliefs questionnaire: & & \\
\hline Work (0-42 points) & $13.7(9.83)$ & $14.7(10.10)$ \\
\hline Physical activities (0-24 points) & $12.7(5.47)$ & $13.8(5.26)$ \\
\hline EuroQoL health index (0-1 point) & $0.73(0.15)$ & $0.71(0.16)$ \\
\hline
\end{tabular}

Table 2 Changes in back pain scores from baseline values in intervention and control groups at 6 weeks, 6 months, and 1 year follow up

\begin{tabular}{|c|c|c|c|c|}
\hline \multirow[b]{2}{*}{ Outcome measure } & \multicolumn{2}{|c|}{$\begin{array}{l}\text { Mean change in } \\
\text { scores* }\end{array}$} & \multirow[b]{2}{*}{ Difference $(95 \%$ Cl) } & \multirow[b]{2}{*}{$\begin{array}{c}P \\
\text { value }\end{array}$} \\
\hline & $\begin{array}{c}\text { Control } \\
\text { group }\end{array}$ & $\begin{array}{l}\text { Intervention } \\
\text { group }\end{array}$ & & \\
\hline At 6 weeks & $(n=94)$ & $(n=85)$ & & \\
\hline Roland disability questionnaire & -1.94 & -2.86 & $0.92(-0.02$ to 1.87$)$ & 0.06 \\
\hline Aberdeen back pain scale & -8.99 & -11.58 & $2.59(-0.37$ to 5.55$)$ & 0.09 \\
\hline Pain diary: & $(n=89)$ & $(n=82)$ & & \\
\hline Strength & -9.4 & -12.2 & $2.8(-1.67$ to 7.30$)$ & 0.22 \\
\hline Distress & -5.0 & -10.2 & $5.13(0.41$ to 9.85$)$ & 0.03 \\
\hline \multicolumn{5}{|l|}{ Fear and avoidance beliefs questionnaire: } \\
\hline Work & -1.26 & -2.98 & $1.72(-0.34$ to 3.78$)$ & 0.10 \\
\hline Physical activities & -2.02 & -3.26 & $1.24(-0.27$ to 2.74$)$ & 0.11 \\
\hline EuroQoL health index & 0.022 & 0.030 & $-0.01(-0.09$ to 0.07$)$ & 0.84 \\
\hline $\begin{array}{l}\text { Mean (range) number of visits to general } \\
\text { practitioner }\end{array}$ & $0.41(0-6)$ & $0.20(0-3)$ & NA & 0.09 \\
\hline At 6 months & $(n=86)$ & $(\mathrm{n}=77)$ & & \\
\hline Roland disability questionnaire & -1.64 & -2.99 & 1.35 (0.13 to 2.57$)$ & 0.03 \\
\hline Aberdeen back pain scale & -8.11 & -10.26 & $2.15(-1.63$ to 5.93$)$ & 0.26 \\
\hline EuroQoL health index & 0.067 & 0.080 & -0.01 (0.06 to 0.04 & 0.60 \\
\hline $\begin{array}{l}\text { Mean (range) number of visits to general } \\
\text { practitioner }\end{array}$ & $0.89(0-8)$ & $0.49(0-4)$ & NA & \\
\hline At 1 year & $(n=88)$ & $(n=83)$ & & \\
\hline Roland disability questionnaire & -1.77 & -3.19 & $1.42(0.29$ to 2.56$)$ & 0.02 \\
\hline Aberdeen back pain scale & -8.48 & -12.92 & 4.44 (1.01 to 7.87$)$ & 0.01 \\
\hline EuroQoL health index & 0.089 & 0.111 & $-0.02(-0.08$ to 0.04$)$ & 0.47 \\
\hline
\end{tabular}

*Adjusted for baseline scores. NA=Not applicable. 
Table 3 Use of services and their costs associated with back pain in the two study groups at 12 months follow up

\begin{tabular}{|c|c|c|c|}
\hline Variable & $\begin{array}{l}\text { Intervention group } \\
(\mathrm{n}=70)^{*}\end{array}$ & $\begin{array}{l}\text { Control group } \\
(n=74)^{\star}\end{array}$ & $\begin{array}{l}\text { Difference }(95 \% \mathrm{CI}) \\
\text { (Student's } t \text { test) }\end{array}$ \\
\hline \multicolumn{4}{|l|}{ Health services } \\
\hline No of exercise classes: & 70 & 0 & \\
\hline $\begin{array}{l}\text { Total cost ( } £ 25.20 \text { per person per } \\
\text { programme of } 8 \text { classes) }\end{array}$ & $£ 1764$ & 0 & \\
\hline No of visits to general practitioner: & 139 & 266 & \\
\hline Total cost (£16 per visit) & $£ 2224$ & $£ 4256$ & \\
\hline No of visits to a physiotherapist: & 65 & 146 & \\
\hline Total cost (£18 per visit) & $£ 1170$ & $£ 2628$ & \\
\hline $\begin{array}{l}\text { No of visits to a chiropractor or } \\
\text { osteopath: }\end{array}$ & 27 & 25 & \\
\hline Total cost (£20 per visit) & $£ 540$ & $£ 500$ & \\
\hline No of visits to orthopaedic surgeon: & 0 & 1 & \\
\hline Total cost (£174 per visit) & 0 & $£ 174$ & \\
\hline No of MRI investigations: & 1 & 1 & \\
\hline Total cost (£300 per visit) & $£ 300$ & $£ 300$ & \\
\hline No of $x$ ray investigations: & 4 & 3 & \\
\hline Total cost (£20 per visit) & $£ 80$ & $£ 60$ & \\
\hline No of nights in hospital: & 0 & 2 & \\
\hline Total cost (£150 per night) & 0 & $£ 300$ & \\
\hline Total health service related costs & $£ 6078$ & $£ 8218$ & \\
\hline Mean (SD) cost per patient & $\begin{array}{c}£ 86.83 \\
(105.19)\end{array}$ & $\begin{array}{l}£ 111.05 \\
(205.11)\end{array}$ & $\begin{array}{l}24.23(-29.94 \text { to } \\
78.39)(P=0.38 \dagger)\end{array}$ \\
\hline Median ( $90 \%$ range) cost per patient & $\begin{array}{c}£ 41.20 \\
(25.20-353.20)\end{array}$ & $0(0-532.00)$ & \\
\hline \multicolumn{4}{|c|}{ Equipment (beds, stove modification, car seat) } \\
\hline No of pieces of equipment: & 4 & 4 & \\
\hline Total cost (item costs $£ 10-800$ ) & $£ 2123$ & $£ 2091$ & \\
\hline \multicolumn{4}{|l|}{ Days off work } \\
\hline Total No of days off work: & 378 & 607 & \\
\hline Total cost (£45 per day)‡ & $£ 17010$ & $£ 27315$ & \\
\hline \multicolumn{4}{|c|}{ All costs (including equipment and days off work) } \\
\hline Total costs & $£ 25211$ & $£ 37624$ & \\
\hline Mean (SD) cost per patient & $\begin{array}{l}£ 360.15 \\
(582.27)\end{array}$ & $\begin{array}{c}£ 508.43 \\
(1108.79)\end{array}$ & $\begin{array}{l}148.28(-145.92 \text { to } \\
442.48)(P=0.32 \S)\end{array}$ \\
\hline Median ( $90 \%$ range) cost per patient & $\begin{array}{c}£ 115.20 \\
(25.20-1688.40)\end{array}$ & $\begin{array}{c}£ 50.00 \\
(0-2728.00)\end{array}$ & \\
\hline
\end{tabular}

MRI=magnetic resonance imaging

*Based on 144 subjects without missing data on resource use or costs.

†Bootstrap comparison of means $\mathrm{P}=0.38$, accelerated $95 \% \mathrm{Cl}$ corrected for bias ( -22.00 to 81.90$)$.

$\ddagger$ Based on gross domestic product capita per head 1996.

$\S B$ Botstrap comparison of means $\mathrm{P}=0.33$, accelerated $95 \% \mathrm{Cl}$ corrected for bias (-89.78 to 506.14 )

done so at six weeks, $60 \%(49 \%$ to $71 \%)$ at six months, and $64 \%(54 \%$ to $74 \%)$ at one year. A smaller proportion of the control group achieved this clinically important improvement: $31 \%(22 \%$ to $40 \%)$ at six weeks, $40 \%(29 \%$ to $50 \%)$ at six months, and $35 \%(25 \%$ to $45 \%)$ at one year.

\section{Patients' preference}

We examined the effect of patients' baseline preference for treatment on outcome after adjusting for baseline scores and main effects. Preference did not significantly affect response to treatment. The intervention had similar effects on both costs and outcomes regardless of baseline preference. For example, the change in the Roland disability questionnaire score at 12 months in the control group was -1.93 for patients who preferred intervention and -1.18 for those who were indifferent $(95 \%$ confidence interval of difference -1.05 to 2.55 ), and in the intervention group the change in score was -3.10 for those who preferred intervention and -3.15 for those who were indifferent ((95\% confidence interval of difference -1.47 to 3.08$)$. As the interaction term (preference by random alloca- tion) was non-significant, the results shown in table 2 exclude the preference term.

\section{Economic evaluation}

Patients in the intervention group tended to use fewer healthcare and other resources compared with those in the control group (table 3). However, the mean difference, totalling $£ 148$ per patient, was not significant: the $95 \%$ confidence interval suggests there could have been a saving of as much as $£ 442$ per patient in the intervention group or an additional cost of up to $£ 146$. Patients in the control group took a total of 607 days off work during the 12 months after randomisation compared with 378 days taken off by the intervention group.

\section{Discussion}

Our results support the hypothesis that a simple exercise class can lead to long term improvements for back pain sufferers. Studies have shown that a similar programme for patients with chronic back pain can be effective in the hospital setting. ${ }^{6}$ In this study we show the clinical effectiveness for patients with subacute or recurrent low back pain who were referred by their general practitioner to a community programme.

Current management guidelines for low back pain recommend a return to physical activity and taking exercise. In particular, they recommend that patients who are not improving at six weeks after onset of back pain, which may be a higher proportion than previously realised, ${ }^{1}$ should be referred to a reactivation programme. The programme we evaluated fits that requirement well. It shows participants how they can safely start moving again and increase their levels of physical activity. It is simple and less costly than individual treatment.

It seemed to have beneficial effects even one year later, as measured by functional disability (Roland disability questionnaire) and clinical status (Aberdeen back pain scale). The mean changes in scores on these instruments were small, with many patients reporting mild symptoms on the day of entry to the trial. However, a substantially larger proportion of participants in the exercise classes gained increases of over three points on the Roland disability questionnaire at six weeks, six months, and one year, which might be clinically important. At six weeks, participants in the exercise classes reported significantly less distressing pain compared with the control group, although the intensity of pain was not significantly different. This is consistent with findings from a study of chronic back pain patients in Oxford, in which changes in distressing pain were much greater than were the changes in intensity of pain. ${ }^{6}$

People with back pain who use coping strategies that do not avoid movement and pain have less disability. ${ }^{18-22}$ In our study the participants in the exercise classes were able to function better according to Roland disability questionnaire scores than the control group at six months and one year after randomisation to treatment, and at one year they also showed a significantly greater improvement in clinical status as measured by the Aberdeen back pain scale. This increase in differences in effect between the intervention and control groups over time is consistent with the results from long term follow up in comparable back pain trials. ${ }^{23}$ 
Health Sciences and Clinical Evaluation, University of York) advised on the statistical analysis. Of the 87 general practitioners who agreed to take part, most referred patients to the study and Drs Allan Harris, Trevor Julian, and John Bush facilitated recruitment of patients while Gillian Rodriguez and Michael White helped to set up exercise classes during the pilot phase. The patients participating in the trial responded to questionnaires at baseline and follow up. Vanessa Waby provided administrative support.JKM is guarantor for the study.

Funding: This research was funded by the Arthritis Research Campaign, the Northern and Yorkshire Regional Health Authority, and the National Back Pain Association.

Competing interests: None declared. to cope better with their pain and function better even one year later

- Patients' preferences for type of management did not affect outcome

- Patients in the intervention group tended to use fewer healthcare resources and took fewer days off work

- This type of exercise programme should be more widely available

\section{Study design}

The design of this study was a conventional randomised controlled trial in that all eligible patients were randomised. However, the participants were asked to state their preferred treatment before they knew of their allocation. A study of antenatal services showed that preferences can be an important determinant of outcome,${ }^{10}$ but we did not find any strong effect of preference on the outcome, although a much larger sample size would be needed to confidently exclude any modest interaction between preference and outcome. ${ }^{8}$ This information may be useful to clinicians in that it suggests that exercise classes are effective even in patients who are not highly motivated. Our trial design, of asking patients for their preferences at the outset, has substantial advantages over the usual patient preference design, in which costs and outcomes cannot be reliably controlled for confounding by preference.

\section{Conclusions}

Our exercise programme did not seem to influence the intensity of pain but did affect the participants' ability to cope with the pain in the short term and even more so in the longer term. It used a cognitive-behavioural model, shifting the emphasis away from a disease model to a model of normal human behaviour, and with minimal extra training a physiotherapist can run it. Patients' preferences did not seem to influence the outcome.

Contributors: JKM conceived the study, developed the protocol, obtained funding, designed and directed the trial, recruited general practitioners, and drafted the paper. DT advised on the organisation and collection of economic data, carried out statistical analysis of the costs, and helped to write the paper. SB-S coordinated the study, assisted in its planning and data collection, and commented on early drafts of the paper. DJ and HL-P assisted in planning the trial and data collection, acted as exercise class leaders, and commented on early drafts of the paper. AF carried out the statistical analysis and helped to draft the paper.JB used the "bootstrapping" technique to help analysis of costs and commented on drafts of the paper. Trevor Sheldon and Alan Maynard (Centre for Health Economics, University of York) and Anthony Dowell (Centre for Primary Care Research, University of Leeds) helped develop the protocol and obtain funding. Trevor Sheldon provided further methodological support and advice. Ian Russell (Department of
Croft P, ed. Low back pain. Oxford: Radcliffe Medical Press, 1997.

2 Clinical Standards Advisory Group. Back pain. London: HMSO, 1994

3 Waddell G, Feder G, McIntosh A, Lewis M, Hutchinson A. Low back pain evidence review. London: Roval College of General Practitioners, 1996.

4 Malmivaara A, Hakkinen U, Aro T, Heinrichs M, Koskenniemi L, Kuosma $\mathrm{E}$, et al. The treatment of acute low back pain-bed rest, exercises or ordinary activity? N Engl J Med 1995;332:351-5.

5 Faas A, Chavannes A, van Eijk JTM, Gubbels J. A randomized, placebo-controlled trial of exercise therapy in patients with acute low back pain. Spine 1993;18:1388-95.

6 Frost H, Klaber Moffett J, Moser J, Fairbank J. Evaluation of a fitness programme for patients with chronic low back pain. BMJ 1995;310:151-4 programme for patients with chronic low back pain: 2 year follow-up of a randomised controlled trial. Pain 1998;75:273-9.

8 McPherson K, Britton A, Wennberg J. Are randomised controlled trials controlled? Patient preferences and unblind trials. $J R$ Soc Med 1997;90:652-6.

9 Bradley C. Designing medical and educational studies. Diabetes Care 1993;16:509-18.

10 Clement S, Sikorski J, Wilson J, Candy B. Merits of alternative strategies for incorporating patient preferences into clinical trials must be considered carefully [letter]. BMJ 1998;317:78

11 Torgerson D, Klaber Moffett J, Russell I. Patient preferences in 1996;1(4):194-7.

12 Roland M, Morris R. A study of the natural causes of back pain. Part 1 : Development of a reliable and sensitive measure of disability in low-back pain. Spine 1983;8:141-4.

13 Ruta D, Garratt A, Wardlaw D, Russell I. Developing a valid and reliable measure of health outcome for patients with low back pain. Spine 1994;19:1887-96.

14 Brooks R with EuroQoL Group. EuroQoL: the current state of play. Health Policy 1996;37:53-72.

15 Waddell G, Newton M, Henderson I, Somerville D, Main C. A fear-avoidance beliefs questionnaire (FABQ) and the role of fearavoidance beliefs in chronic low back pain and disability. Pain 1993;52:157-68

16 Jensen M, McFarland C. Increasing the reliability and validity of pain intensity measurement in chronic pain patients. Pain 1993;55:195-203.

17 Efron B, Tibshirani R. An introduction to bootstrap. New York: Chapman and Hall, 1993.

18 Williams D, Keefe F. Pain beliefs and use of cognitive-behavioral coping strategies. Pain 1991;46:185-90.

19 Estlander A, Harkapaa K. Relationships between coping strategies, disability and pain levels in patients with chronic low back pain. Scand J Behav Ther 1989;18:56-69.

20 Holmes J, Stevenson C. Differential effects of avoidant and attentiona coping strategies on adaption to chronic and recent-onset pain. Health Psychology 1990;9:577-84.

21 Rosenstiel A, Keefe F. The use of coping strategies in chronic low back pain patients: relationship to patient characteristics and current adjustments. Pain 1983;17:33-44

22 Slade P, Troup J, Lethem J, Bentley G. The fear avoidance model of exagpain. Behav Res Ther 1983;21:409-16.

23 Meade T, Dyer S, Browne W, Frank A. Randomised comparison of chiropractic and hospital outpatient management for low back pain: results from extended follow up. BMJ 1995:311:349-51.

24 Cherkin D, Deyo R, Battie M, Street J, Barlow W. A comparison of physical therapy, chiropractic manipulation, and provision of an educational booklet for the treatment of patients with low back pain. $N$ Engl J Med 1998;339:1021-9.

(Accepted 20 May 1999)

\section{Endpiece \\ Forever in his debt}

What one can say with conviction is that, even if every idea which Freud put forward could be proven wrong, we should still be greatly in his debt.

Anthony Storr, Freud (1989)
7 Frost H, Lamb S, Klaber Moffett J, Fairbank J, Moser J. A fitness randomised trials: threat or opportunity? J Health Serv Res Policy gerated pain perception II Preliminary studies of coping strategies for 